\title{
Predicting Malignancy by Peroral Pancreatoscopy of an Intraductal Papillary Mucinous Neoplasm with a Dilated Main Pancreatic Duct: Is Seeing Enough?
}

\author{
Yun Nah Lee and Jong Ho Moon \\ Digestive Disease Center and Research Institute, Department of Internal Medicine, SoonChunHyang University School of Medicine, \\ Bucheon and Cheonan, Korea
}

See "Peroral Pancreatoscopy with Videoscopy and Narrow-Band Imaging in Intraductal Papillary Mucinous Neoplasms with Dilatation of the Main Pancreatic Duct” by Yui Kishimoto, Naoki Okano, Ken Ito, et al., on page 270-278. Clin Endosc 2022;55:213-214

Intraductal papillary mucinous neoplasm (IPMN) of the pancreas is subdivided based on the site of involvement within the main pancreatic duct (MPD) as follows: the main duct (MD), the branch duct, or a mixture of the two. Surgical resection is strongly recommended for MD-IPMNs because of their high malignant potential. In a previous surgical series, the malignancy rate of MD-IPMN was $36-100 \%$. However, this may be an overestimation of the actual risk. Surgical series can present selection bias, as patients who undergo surgical resection are more likely to present with criteria suspicious for progression.

The international consensus guidelines for managing IPMN recommend resecting all MD-IPMNs with an MPD diameter $>10 \mathrm{~mm}$, jaundice, or mural nodules. ${ }^{1}$ Mural nodules have been established as a predictor of malignancy in MD-IPMNs, with high sensitivity (59-92\%) and specificity (60-96\%). ${ }^{2-4}$ However, all MD-IPMNs are not diagnosed to be malignant, despite being surgically resected using these criteria. In the

Received: February 2, 2022 Revised: February 8, 2022

Accepted: February 9, 2022

Correspondence: Jong Ho Moon

Digestive Disease Center and Research Institute, SoonChunHyang University Bucheon Hospital, 170 Jomaru-ro, Wonmi-gu, Bucheon 14584, Korea

Tel: +82-32-621-5094, Fax: +82-32-621-5080, E-mail: jhmoon@schmc.ac.kr

ORCID: https://orcid.org/0000-0002-3946-9944

(c) This is an Open Access article distributed under the terms of the Creative Commons Attribution Non-Commercial License (http://creativecommons.org/ licenses/by-nc/3.0) which permits unrestricted non-commercial use, distribution, and reproduction in any medium, provided the original work is properly cited. current issue of Clinical Endoscopy, Kishimoto et al. ${ }^{5}$ reported the efficacy of endoscopic findings from peroral pancreatoscopy (POP) with video and narrow-band imaging (NBI) for mural nodules to predict the progression of IPMNs with a dilated MPD. They classified mural nodules into four types based on the morphology of protrusion, using POP. Cytology/ biopsy was also performed during POP. Malignancy was not diagnosed by cytology/biopsy in any of the seven patients with mural nodules of sessile morphology. They underwent surveillance only, without surgical resection, and adenocarcinoma developed in one patient (14\%) during the follow-up period (mean: 81 months). The authors suggested that a benign or malignant diagnosis is possible in MD-IPMN depending on the degree of elevation of the mural nodule observed by POP.

With remarkable technological innovation and progress in endoscopic equipment, endoscopic morphology with image-enhanced endoscopy provides high diagnostic yields and has replaced pathological diagnosis for stomach and colon tumors. Recently, peroral cholangiopancreatoscopy (POCP) has shown notable development in terms of technical difficulty and image quality. Subsequently, the diagnostic and therapeutic roles of POCP have continued to expand and evolve. One of the popular indications for peroral cholangioscopy (POC) is the visual diagnosis of indeterminate biliary strictures. In previous studies, visual assessment using POC has shown high sensitivity (78-95\%) and high specificity (73-96\%) for the diagnosis of malignant biliary stricture. ${ }^{6-9}$ Furthermore, image-enhanced technologies such as NBI and I-scan are use- 
ful tools to enhance the diagnostic capability of POP in mural nodules. This is because it is helpful for the observation of fine mucosal structures and tumor vessels compared with whitelight images. Therefore, POP with NBI also has the potential to be effective in predicting the malignant potential of mural nodules in MD-IPMNs, which helps reduce unnecessary surgical resections in MD-IPMNs with mural nodules.

However, malignant progression of MD-IPMN cannot be determined simply by the presence of a mural nodule. Other risk factors, such as MPD diameter, clinical symptoms, and age, should be considered before deciding on surgical resection. Moreover, the natural history of cancer progression in MD-IPMN has not been well characterized. Thus, it is unknown for how long, how often, and which modalities are adaptable for the surveillance of MD-IPMN with a sessile mural nodule. Can MD-IPMNs be observed without resection when mural nodules with sessile morphology are diagnosed by POP in a young patient with no risk for surgery? Although these patients could well be free from adverse events of pancreatic surgery, they would live with a $14 \%$ chance of malignancy for the rest of their lives.

Nevertheless, efforts to predict malignancy in patients with MD-IPMN are worthwhile. To date, there has been no single highly accurate predictive factor for MD-IPMN malignancy. Therefore, risk stratification using several predictive factors is required to improve the diagnostic accuracy for MD-IPMN malignancies. A recent study reported that mural nodules of size $>10 \mathrm{~mm}$ and positive cytology are independent predictors of malignancy in MD-IPMN. ${ }^{10}$ Thus, a study on the correlation between POP findings and mural nodule size is likely to be valuable and interesting.

MD-IPMN has a high risk of progression to malignancy as it always exhibits low-grade dysplasia and should be considered premalignant in all clinical situations. The primary goal of managing MD-IPMN is to treat any associated invasive component and prevent malignancy, while avoiding unnecessary pancreatic resection. In addition, surgical risk should be considered along with patient age and comorbidities. Therefore, it is important to identify the predictors of malignancy in MD-IPMNs to help with decisions regarding surgical resection. Although the current study is a first step, and further prospective studies are needed, endoscopic classification of protrusions for the mural nodules of MD-IPMN may have clinical significance in predicting malignant potential, and there is great anticipation in the future.
Conflicts of Interest

The authors have no potential conflicts of interest.

Funding

None.

Author Contributions

Conceptualization: Yun Nah Lee

Project administration: Jong Ho Moon

ORCID

Yun Nah Lee

Jong Ho Moon

https://orcid.org/0000-0001-5588-784X

https://orcid.org/0000-0002-3946-9944

\section{REFERENCES}

1. Tanaka M, Fernández-Del Castillo C, Kamisawa T, et al. Revisions of international consensus Fukuoka guidelines for the management of IPMN of the pancreas. Pancreatology 2017;17:738-753.

2. Takuma K, Kamisawa T, Anjiki H, et al. Predictors of malignancy and natural history of main-duct intraductal papillary mucinous neoplasms of the pancreas. Pancreas 2011;40:371-375.

3. Serikawa M, Sasaki T, Fujimoto Y, Kuwahara K, Chayama K. Management of intraductal papillary-mucinous neoplasm of the pancreas: treatment strategy based on morphologic classification. J Clin Gastroenterol 2006;40:856-862.

4. Manfredi R, Graziani R, Motton M, et al. Main pancreatic duct intraductal papillary mucinous neoplasms: accuracy of MR imaging in differentiation between benign and malignant tumors compared with histopathologic analysis. Radiology 2009;253:106-115.

5. Kishimoto Y, Okano N, Ito K, et al. Peroral pancreatoscopy with videoscopy and narrow-band imaging in intraductal papillary mucinous neoplasms with dilatation of the main pancreatic duct. Clin Endosc 2022;55:270-278

6. Ramchandani M, Reddy DN, Gupta R, et al. Role of single-operator peroral cholangioscopy in the diagnosis of indeterminate biliary lesions: a single-center, prospective study. Gastrointest Endosc 2011;74:511-519.

7. Varadarajulu S, Bang JY, Hasan MK, Navaneethan U, Hawes R, Hebert-Magee S. Improving the diagnostic yield of single-operator cholangioscopy-guided biopsy of indeterminate biliary strictures: ROSE to the rescue? (with video). Gastrointest Endosc 2016;84:681-687.

8. Lee YN, Moon JH, Choi HJ, et al. Direct peroral cholangioscopy for diagnosis of bile duct lesions using an I-SCAN ultraslim endoscope: a pilot study. Endoscopy 2017;49:675-681.

9. Mounzer R, Austin GL, Wani S, Brauer BC, Fukami N, Shah RJ. Per-oral video cholangiopancreatoscopy with narrow-band imaging for the evaluation of indeterminate pancreaticobiliary disease. Gastrointest Endosc 2017;85:509-517.

10. Uehara H, Abe Y, Kai Y, et al. Predictors of malignancy in main duct intraductal papillary mucinous neoplasm of the pancreas. Gastrointest Endosc 2022;95:291-296 